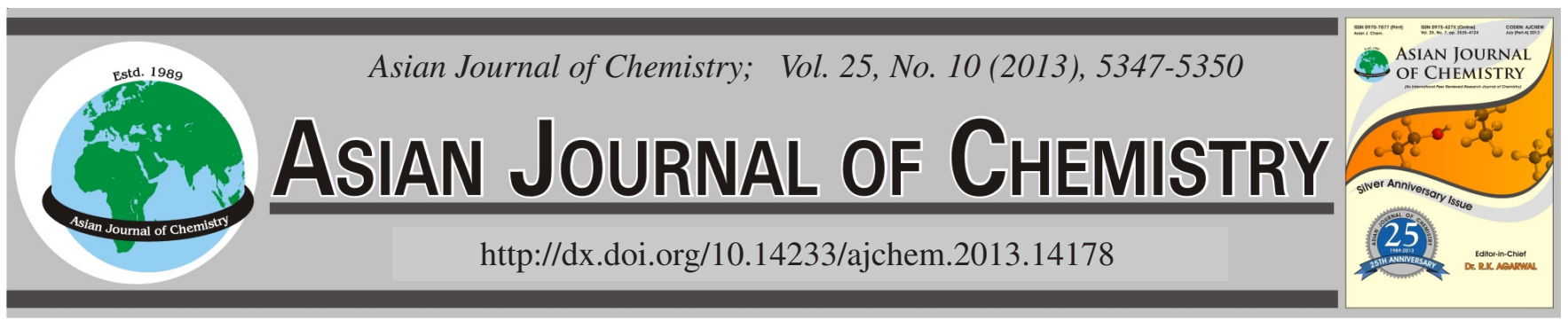

\title{
Synthesis and Characterization of Film-forming Polymer/SiO Nanocomposite via Surfactant-Free Emulsion Polymerization
}

\author{
Qianqian Shang, Hu Liu, LiJing Gao and GuOmin XiaO*
}

School of Chemistry and Chemical Engineering, Southeast University, Nanjing 211189, P.R. China

*Corresponding author: Tel/Fax: +86 25 52090612; E-mail: xiaogm@ seu.edu.cn

Poly(methyl methacrylate-co-butyl acrylate-co-methacrylic acid)/SiO $\left.\left(\mathrm{PMMA}_{2} \mathrm{BA}-\mathrm{MAA}\right) / \mathrm{SiO}_{2}\right)$ nanocomposite particles were prepared via surfactant-free emulsion polymerization. The effects of $\mathrm{SiO}_{2}$ content on the emulsion stability, monomer conversion and polymerization rate were investigated. The experimental results revealed that (PMMA-BA-MAA)/ $\mathrm{SiO}_{2}$ nanocomposite particles were film-forming and the polymer spheres with ordered arrangement structure were bounded by $\mathrm{SiO}_{2}$ networks. $\mathrm{SiO}_{2}$ particles (or aggregates), which had strong interaction with the polymer matrix, were enriched at the surface of the nanocomposite films. The dried nanocomposite films showed better water resistance than the pure polymer film and the film containing $10 \mathrm{wt} \% \mathrm{SiO}_{2}$ had the lowest water adsorption $(0.070 \mathrm{~g} / \mathrm{g})$.

Key Words: Film-forming, Nanocomposite, Emulsion polymerization, $\mathrm{SiO}_{2}$ particles, Acrylic polymer.

\section{INTRODUCTION}

The field of organic/inorganic nanocomposite materials is of growing interest, because they combine the properties of the polymer moiety with inorganic nanoparticles in an advantageous way, e.g., amalgamating the flexibility, the toughness and the ability of the polymer to form films with the thermal stability and the mechanical strength of the inorganic nanoparticles ${ }^{1-6}$. These materials are likely to become one of the fastest developing materials because of their extensive potential applications in various fields of material science, ranging from nanolithography ${ }^{2}$, next-generation exterior facade paints ${ }^{3}$ and scratch-resistant transparent coatings ${ }^{4}$ to polymer light-emitting diodes ${ }^{5}$ and molecular imprinting ${ }^{6}$.

In the past decades, many methods have been developed to prepare organic/inorganic nanocomposite materials, such as sol-gel process $^{7,8}$, free-radical copolymerization ${ }^{9,10}$, emulsion polymerization ${ }^{11-15}$ and self-assembly methods ${ }^{16}$. Among these methods, emulsion polymerization has become the most frequently used technology, since its easy synthesis processes and good stability of polymer nanocomposite latex particles. An enormous variety of inorganic particles have been associated to nanocomposite materials, among which silica was the most intensively studied due to its high stability, high thermal resistance, electrical and optic properties, as well as its extensive applications in diverse areas ${ }^{17}$. Recently, many studies have been devoted to the preparation of organic/ inorganic nanocomposites in the presence of $\mathrm{SiO}_{2}$ particles.
For example, Qiao et al. ${ }^{18}$, presented a facile preparation approach for raspberry-like silica/polystyrene/silica multilayer nanocomposite particles via mini-emulsion polymerization. They used larger surface-modified $\mathrm{SiO}_{2}$ as the core and smaller unmodified $\mathrm{SiO}_{2}$ as the shell with 4-vinylpyridine as an auxiliary monomer. Guo et al. ${ }^{19}$, prepared MPS-nanosilica/acrylic core-shell composite latex and discussed its application in toughening PVC matrix. However, it seemed that the majority of these works were focused on the preparation of nanocomposites with the functional modified inorganic particles as the core in the presence of surfactant and only a few works were involved in the formation of nanocomposites by surfactantfree emulsion polymerization and the effect of the unmodified inorganic particles on the kinetics of the surfactant-free emulsion polymerization systems.

In this work, we introduced a novel method for the synthesis of film-forming poly(methyl methacrylate-co-butyl acrylate-co-methacrylic acid)/SiO $(\mathrm{PMMA-BA-MAA}) / \mathrm{SiO}_{2}$ ) nanocomposite particles through surfactant-free emulsion polymerization with an ordered arrangement surface structure. The effects of $\mathrm{SiO}_{2}$ content on the emulsion stability, monomer conversion and polymerization rate were investigated. The nanocomposite particles were dried to form nanocomposite films and the water resistance of the films was investigated.

\section{EXPERIMENTAL}

Tetraethoxysilane (TEOS), methyl methacrylate (MMA), butyl acrylate (BA), methacrylic acid (MAA) and ammonium 
persulfate (APS) were purchased from Shanghai Lingfeng Chemical Reagent CO. Ltd., China. Methyl methacrylate and butyl acrylate were distilled to remove the inhibitor in vacuo and stored at $4{ }^{\circ} \mathrm{C}$ for use. The other materials were used as received. Deionized water was used in all experiments.

Preparation of $\mathrm{SiO}_{2}$ nanoparticles: $\mathrm{SiO}_{2}$ particles were synthesized by Stöber method ${ }^{20}$. The reaction was carried out in a $500 \mathrm{~mL}$ three-neck round-bottom flask with a mixture of $300 \mathrm{~mL}$ ethanol and $25 \mathrm{~mL}$ aqueous solution of ammonia. The mixture was stirred vigorously to homogenize at room temperature. After stabilization, tetraethoxysilane $(10 \mathrm{~mL})$ was quickly added into the solution and the hydrolysis and condensation reaction occurred with continuous stirring for $36 \mathrm{~h}$. The obtained $\mathrm{SiO}_{2}$ particles were purified by centrifugation, decantation and resuspension in water with ultrasonic for three times.

Synthesis of nanocomposite particles: The nanocomposite particles were synthesized by the copolymerization of MMA, BA and MAA under aqueous conditions in the present of $\mathrm{SiO}_{2}$ nanoparticles. The typical experimental process was as follows: $\mathrm{SiO}_{2}$ particles (0.3 g), MMA(7.2 g), BA (4.8 g) and MAA $(0.232 \mathrm{~g}, 2.7 \mathrm{mmol})$ were mixed with $100 \mathrm{~mL}$ water and then the mixture were placed into a $250 \mathrm{~mL}$ four-necked flask equipped with a reflux condenser, drop ping funnel, nitrogen gas inlet and a mechanical stirrer. After sufficiently degassed under stirring, the reactor was placed in a water bath at $75^{\circ} \mathrm{C}$. Then ammonium persulfate (APS) aqueous solution ( $0.12 \mathrm{~g}$ of APS was dissolved in $8 \mathrm{~mL}$ deionized water) was added to initiate emulsion polymerization. The reaction system was stirred at $75^{\circ} \mathrm{C}$ for $4 \mathrm{~h}$. The samples were taken out of the reactor at regular intervals to determine the monomer conversion after adding a drop of hydroquinone aqueous solution. The latex particles without $\mathrm{SiO}_{2}$ nanoparticles were also prepared by surfactant-free emulsion polymerization using different dosages of MAA.

Fabrication of nanocomposite films: The nanocomposite films were fabricated by drop casting the nanocomposite emulsion on freshly cleaned glass substrates. First, the glass substrates were cleaned in detergent solution by sonication for $0.5 \mathrm{~h}$ and rinsed with water thoroughly. Then, as-prepared nanocomposite emulsion was carefully cast on this cleaned glass substrate and solvent was allowed to evaporate at $80{ }^{\circ} \mathrm{C}$ for $4 \mathrm{~h}$.

Characterization: Monomer conversion (M) and solids concentration $(\mathrm{S})$ were determined using the gravimetric method. Coagulum was filtered using a strainer, washed by water and dried to a constant weight at $80^{\circ} \mathrm{C}$. The dry mass of coagulum $\left(\mathrm{m}_{\mathrm{c}}\right)$ and total emulsion mass $\left(\mathrm{m}_{\mathrm{e}}\right)$ was measured and the coagulum concentration $(\mathrm{C})$ was determined by the mass ratio of $m_{c}$ and $m_{e}$. Fourier-transform infrared analyses were conducted using a Nicolet 5700 Fourier-transform spectrometer in the range of $4000-400 \mathrm{~cm}^{-1}$ at room temperature (Thermo Electron Corporation, America). Scanning electron microscopy (SEM, S-3000N, Hitach Company, Japan) was employed to characterize the morphologies of silica nanoparticles, nanocomposite latex particles and films. Water absorption of the films was determined by measuring the weight variation of films before and after being immerged into deionized water for $24 \mathrm{~h}$ at ambient temperature.

\section{RESULTS AND DISCUSSION}

Synthesis of (PMMA-BA-MAA)/SiO 2 nanocomposite particles: The surfactant-free emulsion polymerization was carried out at various MAA dosages and the effect of MAA on the monomer conversion and coagulum content was shown in Table-1. The coagulum content was used to evaluate the polymerization stability. The larger the value of coagulation content is, the worse the polymerization stability is. Since preliminary experiments without the addition of MAA led to unstable solution, MAA was added into the polymerizations. When 2.1-3.3 mmol of MAA was introduced, the emulsion polymerization processes were stable and the optimal amount of MAA was around $2.7 \mathrm{mmol}$, at which the monomer conversion was higher than $90 \%$ and the coagulum content was lower than $1 \%$. With either less or more MAA, the monomer conversion decreased and the formation of the coagulum occurred. Therefore, we subsequently carried out the surfactant-free emulsion polymerization using $2.7 \mathrm{mmol}$ MAA.

The effects of $\mathrm{SiO}_{2}$ content on the solid content of the emulsion and the monomer conversion was investigated, as shown in Table-2. It could be seen that the solid content of the emulsion and the degree of monomer conversion increased when silica nanoparticles were added into the reaction system. This was because it was of benefit for the formation of hydrogen bonds between the silanol groups of the silica particles and the $-\mathrm{COOH}$ groups on the polymer particles surface under a acidic character of polymerization condition $(\mathrm{pH}=2.5-5.5)^{21}$ via hydrogen bonds, the $\mathrm{SiO}_{2}$ nanoparticles could enhance the stability of latex by providing the polymer latex particles with steric hindrance stabilization.

SEM image of $\mathrm{SiO}_{2}$ nanoparticles was shown Fig. 1, which demonstrated that the as-prepared $\mathrm{SiO}_{2}$ nanoparticles were uniformly spherical and highly monodisperse. The average diameter of $\mathrm{SiO}_{2}$ nanoparticles determined by SEM was 60 $\mathrm{nm}$. The relationship between $\mathrm{SiO}_{2}$ content and the reaction rate was illustrated, as shown in Fig. 2. It was found that the reaction rate was accelerated and the monomer conversion was increased when $\mathrm{SiO}_{2}$ nanoparticles were added. The initiate rate of polymerization in the present of $\mathrm{SiO}_{2}$ particles was faster

TABLE-1

EFFECT OF MAA ON THE SURFACTANT-FREE EMULSION POLYMERIZATION

\begin{tabular}{ccccccccc}
\hline Run & MAA $(\mathrm{mmol})$ & MMA $(\mathrm{g})$ & BA $(\mathrm{g})$ & APS $(\mathrm{g})$ & Water $(\mathrm{g})$ & $\mathrm{S}(\%)$ & M $(\%)$ & C $(\%)$ \\
\hline 1 & 0 & 7.2 & 4.8 & 0.12 & 108 & - & - & Agglomerating \\
2 & 2.1 & 7.2 & 4.8 & 0.12 & 108 & 7.86 & 78.7 & 8.56 \\
3 & 2.4 & 7.2 & 4.8 & 0.12 & 108 & 8.59 & 85.8 & 0.37 \\
4 & 2.7 & 7.2 & 4.8 & 0.12 & 108 & 9.04 & 90.7 & 0.24 \\
5 & 3.0 & 7.2 & 4.8 & 0.12 & 108 & 8.04 & 80.0 & 1.44 \\
6 & 3.3 & 7.2 & 4.8 & 0.12 & 108 & 7.38 & 73.3 & 6.32 \\
\hline
\end{tabular}




\begin{tabular}{ccccccc}
\hline \multicolumn{7}{c}{ TABLE-2 } \\
\multicolumn{7}{c}{ EFFECT OF $\mathrm{SiO}_{2}$ CONTENT ON THE SURFACTANT- } \\
FREE EMULSION POLYMERIZATION
\end{tabular}

${ }^{\mathrm{a}} 7.2 \mathrm{~g}$ MMA and $4.8 \mathrm{~g}$ BA were added in all runs. ${ }^{\mathrm{b}}$ Based on the total monomer weight.

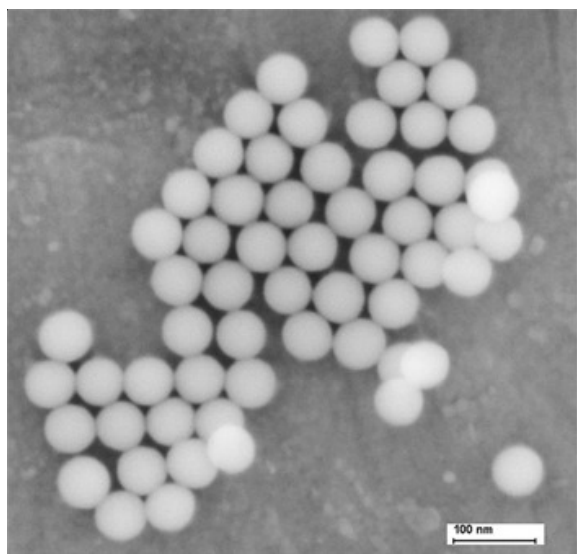

Fig. 1. SEM image of the $\mathrm{SiO}_{2}$ nanoparticles

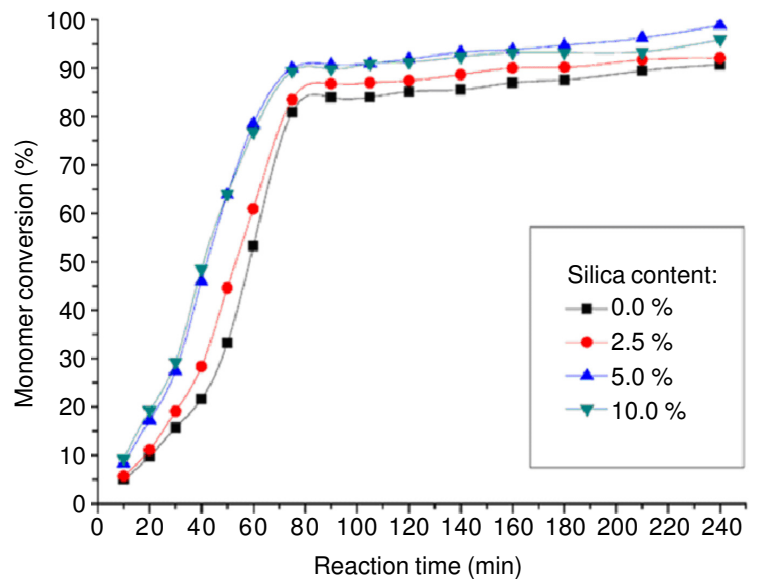

Fig. 2. Effect of $\mathrm{SiO}_{2}$ contents on the conversion versus time curves for emulsifier-free emulsion polymerization

than the system without $\mathrm{SiO}_{2}$ and the reaction rate tended to become greater with the amount of $\mathrm{SiO}_{2}$ up to $10 \mathrm{wt} \%$. However, the monomer conversion was slightly reduced when $\mathrm{SiO}_{2}$ content was higher than $5 \mathrm{wt} \%$, since the nucleation was hindered by the possible aggregation of $\mathrm{SiO}_{2}$ at a high concentration.

Fig. 3 showed the SEM images of the nanocomposite particles with different $\mathrm{SiO}_{2}$ content. The polymer particles without $\mathrm{SiO}_{2}$ were uniformly spherical (Fig. 3a). When $\mathrm{SiO}_{2}$ were introduced into the reaction system, the polymer particles were bounded by $\mathrm{SiO}_{2}$ rather than encapsulated $\mathrm{SiO}_{2}$ particles into interior. At $2.5 \mathrm{wt} \% \mathrm{SiO}_{2}$ particles, superficial and interrelated $\mathrm{SiO}_{2}$ networks were clearly seen on the film surface (Fig. 3b) and the polymer particles were embedded and somewhat orderly arrayed. As $\mathrm{SiO}_{2}$ particles were increased to $5 \mathrm{wt}$ $\%$, smooth ordered film-forming nanocomposite particles
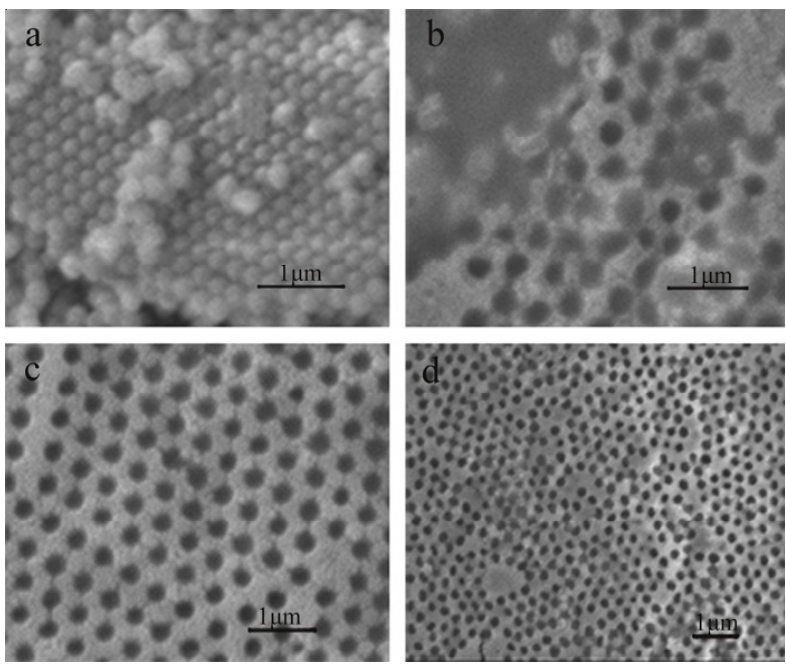

Fig. 3. SEM images for (PMMA-BA-MAA) $/ \mathrm{SiO}_{2}$ nanocomposite particles with different $\mathrm{SiO}_{2}$ contents: a, 0 wt \%; b, 2.5 wt \%; c, 5 wt \%; d, 10 wt $\%$

could be obtained (Fig. 3c). Increasing the $\mathrm{SiO}_{2}$ content up to 10 wt $\%$, the aggregation of $\mathrm{SiO}_{2}$ happened and the nanocomposite latex particles ordered arranged were locally destroyed (Fig. 3d). These results suggested that 5 wt $\% \mathrm{SiO}_{2}$ particles were already enough for the formation of an integrated $\mathrm{SiO}_{2}$ network.

Morphology of (PMMA-BA-MAA)/SiO ${ }_{2}$ nanocomposite films: The typical SEM images of the surface and crosssection of these nanocomposite films were demonstrated in Fig. 4. It could be seen that the surface of the film was rather smooth in the absence of $\mathrm{SiO}_{2}$ particles. However, when the $\mathrm{SiO}_{2}$ content increased to 5 or $10 \mathrm{wt} \%$, particles $c a$. 200-400 $\mathrm{nm}$ brought by agglomeration of the $\mathrm{SiO}_{2}$ particles were observable on the films surface, which indicated the nanocomposite films had silica-rich surfaces. The observation in the film cross-section indicated that the $\mathrm{SiO}_{2}$ particles were implanted into the polymer matrix and kept the continuity and compactness of the film, which meant that there was a strong interaction between the $\mathrm{SiO}_{2}$ particles and the polymer matrix.
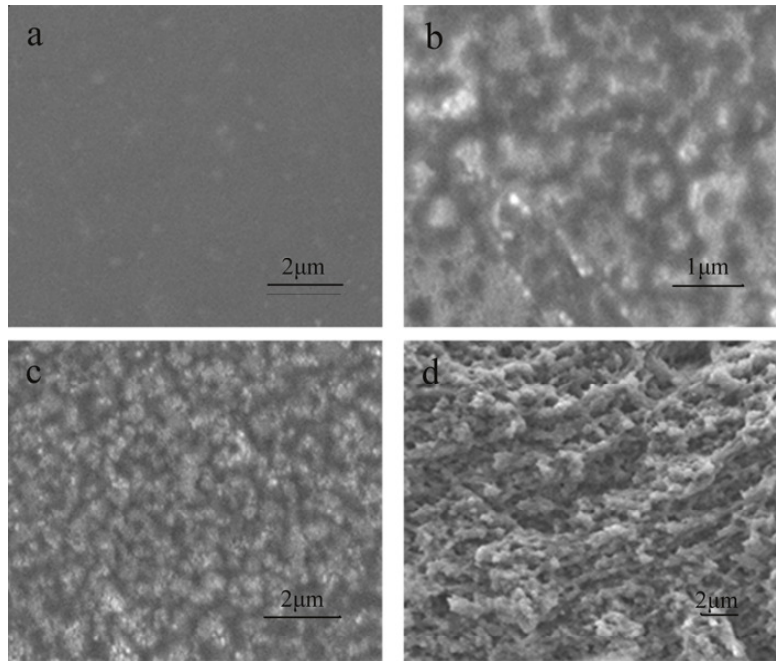

Fig. 4. SEM images of (PMMA-BA-MAA) $/ \mathrm{SiO}_{2}$ nanocomposite films with different $\mathrm{SiO}_{2}$ contents: a, surface, 0 wt \%; b, surface, 5 wt \%; c, surface, 10 wt \%; d, cross-section, $10 \mathrm{wt} \%$ 
The agglomeration of $\mathrm{SiO}_{2}$ particles was mainly caused by the reason that the $\mathrm{SiO}_{2}$ particles surrounded the polymer latex particles rather than within the polymer latex particles (Fig. 3b-d). During the formation stage of the nanocomposite films, these non-embraced $\mathrm{SiO}_{2}$ particles preferentially surrounded polymer latex particles, then contacted with each other and further transformed into $\mathrm{SiO}_{2}$ aggregates via condensation of silanol groups, accompanied with the evaporation of water.

Water resistance of (PMMA-BA-AA)/SiO $\mathbf{S i n}_{2}$ nanocomposite films: Water absorption amount of the nanocomposite films were investigated (Fig. 5). The pure (PMMA-BA-MAA) film had the highest water adsorption $(0.143 \mathrm{~g} / \mathrm{g})$, while nanocomposite films showed lower water adsorption than it. The carboxyl groups on the surface of the pure (PMMA-BAMAA) latex film made the film surface hydrophilic which caused the high water adsorption. In nanocomposite films, $\mathrm{SiO}_{2}$ particles interacted with the polymer chains through hydrogen bonding between the silanol groups and the carbonyl groups and the $\mathrm{SiO}_{2}$ particles aggregated with each other via the interaction of silanol groups. All these decreased the content of hydrophilic portion on the surfaces of nanocomposite films and made the surfaces more hydrophobic. Consequently, the amount of adsorbed water on the film surfaces decreased when the introduced $\mathrm{SiO}_{2}$ particles were increased. The most $\mathrm{SiO}_{2}$ aggregates were detected on the surface of the film containing $10 \mathrm{wt} \% \mathrm{SiO}_{2}$ particles (Fig. 5c); hence the nanocomposite film had the lowest water adsorption $(0.070 \mathrm{~g} / \mathrm{g})$.

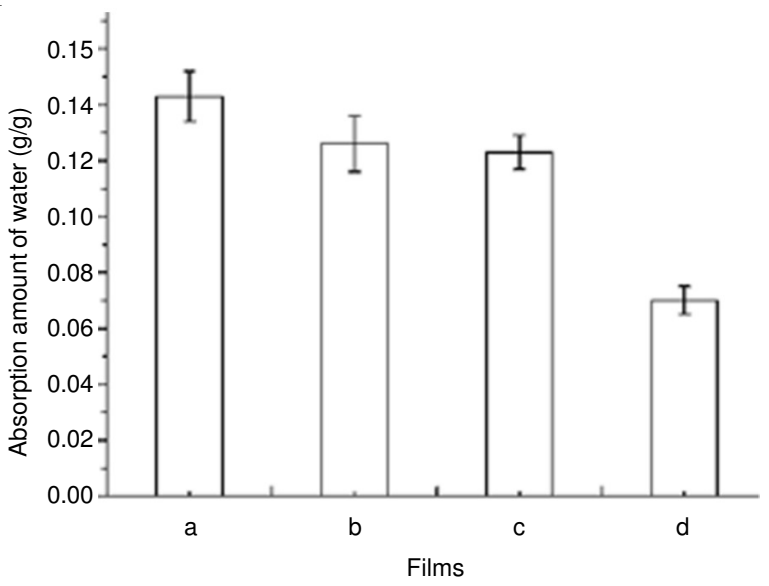

Fig. 5. Absorption amount of water of the nanocomposite films containing various contents of $\mathrm{SiO}_{2}$ particles: a, 0 wt \%; b, $2.5 \mathrm{wt} \%$; c, 5 wt \%; d, 10 wt $\%$

\section{Conclusion}

The facile synthesis of novel film-forming (PMMA-BA$\mathrm{MAA}) / \mathrm{SiO}_{2}$ nanocomposite particles by surfactant-free emulsion polymerization was described in this work. The research results demonstrated that the amount of MAA was very important to the monomer conversion and the $\mathrm{SiO}_{2}$ particles could accelerate the polymerization rate and increase monomer conversion at the dosage from 2.5-10 wt \%. (PMMA-BA$\mathrm{MAA}) / \mathrm{SiO}_{2}$ nanocomposite particles were proved to be filmforming and SEM results indicated the ordered arrangement of polymer spheres bounded by $\mathrm{SiO}_{2}$ networks. Surface and cross-section morphology studies of the nanocomposite films suggested that $\mathrm{SiO}_{2}$ particles were enriched at the film surface and they had strong interaction with the polymer matrix. Water resistance studies indicated that (PMMA-BA-MAA)/ $\mathrm{SiO}_{2}$ nanocomposite films showed better water resistance property compared with the pure polymer film and the amount of adsorbed water decreased along with the increasing of $\mathrm{SiO}_{2}$ content. Thus the (PMMA-BA-MAA)/ $\mathrm{SiO}_{2}$ nanocomposite particles have potential applications in paints and coatings with good water repellency.

\section{ACKNOWLEDGEMENTS}

The authors are grateful for the financial support by the Natural Science Foundation of China (Grants 21076044), the National Basic Research Program of China (973 Program) (Grants 2012CB215306) and the Scientific Research Foundation of Graduate School of Southeast University (GrantYBJJ1223).

\section{REFERENCES}

1. F. Tiarks, K. Landfester and M. Antonietti, Langmuir, 17, 5775 (2001).

2. Y.Q. Hu, H.P. Wu, K. Gonsalves and L. Merhari, Microelectron Eng., 56, 289 (2001).

3. F. Tiarks, J. Leuninger, O. Wagner, E. Jahns and H. Wiese, Surf. Coat. Int., 90, 221 (2007).

4. L. Lewis and D. Katsamberis, J. Appl. Polym. Sci., 42, 1551 (1991).

5. T.W. Lee, O.O. Park, J.Y. Yoon and J.J. Kim, Adv. Mater., 13, 211 (2001).

6. S. Marx and Z. Liron, Chem. Mater., 13, 3624 (2001).

7. M. Sangermano, G. Malucelli, E. Amerio, R. Bongiovanni, A. Priola, A.D. Gianni, B. Voit and G. Rizza, Macromol. Mater. Eng., 291, 517 (2006).

8. X.M. Shi, S.M. Xu, J.T. Lin, S. Feng and J.D. Wang, Mater. Lett., 63, 527 (2009).

9. C.Z. Li and B.C. Benicewicz, Macromolecules, 38, 5929 (2005)

10. P. Liu, W.M. Liu and Q.J. Xue, Eur. Polym. J., 40, 267 (2004).

11. X.F. Ding, J.Z. Zhao, Y.H. Liu, H.B. Zhang and Z.H. Wang, Mater. Lett., 58, 3126 (2004).

12. Y.Q. Wang, Y.P. Li, R.Y. Zhang, L. Huang and W.W. He, Polym. Compos., 27, 282 (2006).

13. L. Yao, T.T. Yang and S.Y. Cheng, J. Appl. Polym. Sci., 115, 3500 (2010).

14. K. Zhang, H.T. Chen, X. Chen, Z.M. Chen, Z.C. Cui and B. Yang, Macromol. Mater. Eng., 288, 380 (2003).

15. J.L. Luna-Xavier, A. Guyot and E. Bourgeat-Lami, J. Colloid Interf. Sci., 250, 82 (2002).

16. C.D. Knorowski, J.A. Anderson and A. Travesset, J. Chem. Phys., 128, 164903 (2008).

17. L.L. Duan, M. Chen, S.X. Zhou and L.M. Wu, Langmuir, 25, 3467 (2009).

18. X.G. Qiao, M. Chen, J. Zhou and L.M. Wu, J. Polym. Sci.-Polym. Chem., 45, 1028 (2007).

19. Y.K. Guo, M.Y. Wang, H.Q. Zhang, G.D. Liu, L.Q. Zhang and X.W. Qu, J. Appl. Polym. Sci., 107, 2671 (2008).

20. W. Stöber, A. Fink and E. Bohn, J. Colloid Interf. Sci., 26, 62 (1968).

21. B.You, N.G.Wen, Y.C. Cao, S.X. Zhou and L.M. Wu, Polym. Int., 58, 519 (2009). 